\title{
First performance measurements of the Fast Tracker Real Time Processor at ATLAS
}

\author{
Nicolò Vladi Biesuz*, on behalf of the ATLAS Collaboration \\ I.N.F.N. sezione di Pisa \\ E-mail: nicolo.vladi.biesuz@cern.ch
}

\begin{abstract}
Real time track reconstruction at hadron colliders plays an important role in selecting interesting events from the huge background while mitigating the effect of pile-up. The Fast Tracker, an upgrade to the current ATLAS trigger system, will feed the High Level Trigger with high quality tracks reconstructed over the entire detector at $100 \mathrm{kHz}$ rate. The Fast Tracker system processes 64 eta-phi towers (partially overlapping), where each tower is processed by a dedicated asynchronous, data-driven pipeline. The combinatorial challenge inherent to tracking is solved with the use of Associative Memory ASICs that compare inner detector hits to millions of pre-calculated patterns simultaneously. The tracking problem within matched patterns is further simplified by using pre-computed linearized fitting constants and leveraging fast digital signal processing in modern commercial FPGAs. Half of the system has been produced and integration in ATLAS is proceeding to demonstrate functionality with real data and partial detector coverage. We show the first results on system performance studies. Tracks were reconstructed and validated with Fast Tracker functional simulation. The system integration in the ATLAS experiment is progressing through 2018 to reach stable track processing. These studies will continue by evaluating the Fast Tracker tracking resolution and latency on real data. Those measurements will allow optimization of the system improving its performance. We report results of this first important experience with data in preparation of the full Fast Tracker operational conditions in 2021.
\end{abstract}

Topical Workshop on Electronics for Particle Physics (TWEPP2018)

17-21 September 2018

Antwerp, Belgium

${ }^{*}$ Speaker. 


\section{Introduction}

The ATLAS detector [2] is a multi-purpose detector operating at the CERN Large Hadron Collider (LHC). The ATLAS detector surrounds the interaction point using a sub-detector structure in order to measure the position and energy of all particles produced in a collision.

During the second LHC run (Run 2), which begun in 2015, the accelerator performance steadily increased. This increase in luminosity meant the experiments operating at the LHC are faced with a growing number of interactions per bunch crossing $(\mu)$. This critical parameter has reached a maximum of $\mu \approx 60$ interactions per bunch crossing in proton-proton collision, averaging at roughly $\langle\mu\rangle=37$. During the next data taking phase foreseen by 2021 (Run 3), the luminosity will be leveled at or above pile-up of $\langle\mu\rangle=60$.

In such a harsh environment it is mandatory to have an efficient trigger system able to reduce the event rate to a sustainable rate retaining only interesting events. The ATLAS experiment uses a two level trigger system [3]. The first trigger level, called Level-1, reduces the event rate from $40 \mathrm{MHz}$ to $100 \mathrm{kHz}$ with a latency of $2.5 \mu \mathrm{s}$ using the data coming from the calorimeters and the muon spectrometer. Data coming from the Inner detector are used only at the next trigger stage, the so called High Level Trigger (HLT). This trigger stage has a latency of $20 \mathrm{~ms}$. Since the track reconstruction for the full detector requires roughly $1 \mathrm{~s} /$ event, at the HLT level tracking is performed only in regions of interest.

The Fast TracKer (FTK) system [4] is a track processor aiming at full exploitation of Inner Detector information.

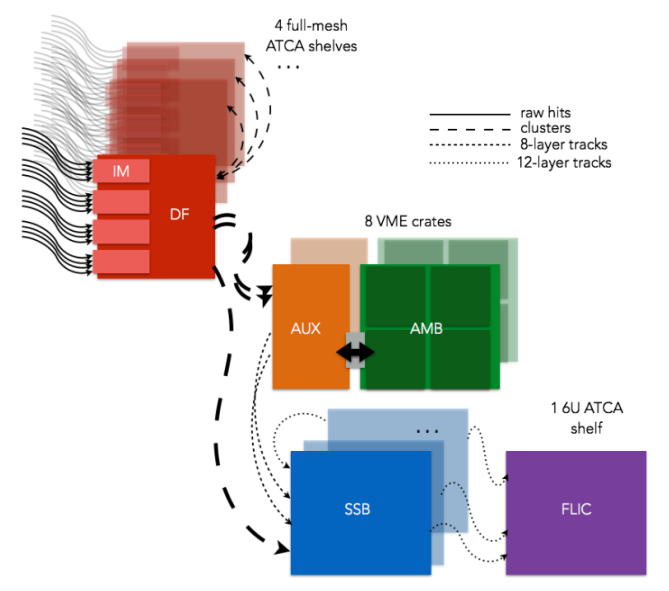

Figure 1: Functional sketch of Fast Tracker system. Electronics boards are represented by the coloured boxes. Arrows indicate dataflow in the system, arrows styles represent different data-processing stages.

\section{The Fast Tracker System}

The Fast Tracker systems, an upgrade to the current ATLAS [3] trigger system, will operate at the first trigger level accept rate of $100 \mathrm{kHz}$. The system will provide high quality tracks reconstructed over the entire detector with an estimated latency of the order of $100 \mu \mathrm{s}$. Therefore these tracks can be used as inputs to the High Level trigger. 
The detector is subdivided into $64 \eta-\phi$ towers each consisting of 12 detector logical layers. Figure 1 shows the FTK functional sketch. Each tower is processed in its own asynchronous, datadriven pipeline. Centroids are sent from the Input Mezzanine (IM) daughter board to the Data Formatters (DF), a motherboard distributing data to the suitable Processing Unit. The Processing Units are composed by the Associative Memory Board [7] (AMB) and the Auxiliary (AUX) Card. The AMB selects the hits to be used to build a track candidate by performing the so called pattern matching detailed in the next section. Hits from the 8-layer track candidates are fitted by the AUX Card. The track candidates whose fit fulfill a loose $\chi^{2}$ quality requirement are sent to the Second Stage Board (SSB). After rejecting duplicates the Second Stage Board extrapolates the track to the remaining detector hits and it fits the complete tracks with 12 layers applying a $\chi^{2}$ cut and determining the helix parameters. These tracks are provided to the HLT through the Interface Card (FLIC).

The final configuration of the system is described in Section 5.

\section{The Fast Tracker algorithm}

The idea behind the FTK operation is to solve the tracking combinatorial challenge performing pattern matching against a set of precalculated trajectories at reduced resolution. Before the data taking phase, the interaction between single particle and the detector is simulated. The obtained detector hits are clustered and converted to a lower resolution suitable for pattern matching. Then the expected detector hit patterns are extracted for each track. Normally more than one simulated track will match a single pattern. The most probable patterns, defined as the ones matching the higher number of tracks, are selected to be stored in an Associative Memory [6].

During the data taking phase hits coming from the detector are compared to the ones stored in the Associative Memory. If a match is found, then the hits that fired the match are retrieved, and they can be used in the fitting procedure. This pattern matching procedure is performed in the AMB with the usage of an ASIC called Associative Memory chip [8]. Roughly 0.5 (1) billion patterns can be stored in the system in the present (final) configuration. The track candidates are validated or rejected with high- resolution Track Fitting [4] using pre-computed linearised fitting constants. The obtained tracks will contain duplicate tracks sharing most, but not all, of their hits. This source of duplicate tracks is removed by keeping only the best $\chi^{2}$ track among the duplicates. The candidates 8 layer tracks are extrapolated to the 4 additional detector layer. The hits closer to the extrapolated track are then used in a 12 detector layer fitting procedure in analogy to the one described.

\section{First Reconstructed Tracks}

Initial FTK prototypes started to be available in 2015 and were used to start the commissioning of the FTK input system. The first AM06 ASICs became available in 2016 allowing to produce the first 8-layer tracks from collisions by the end of 2016. Commissioning up to now focused mostly on two detector towers of the FTK system shown in Figure 2a. Tower 22 is connected to a complete pipeline producing 12-layer tracks while tower 40 uses a reduced version without the SSB and FLIC providing 8-layer tracks. In this proceedings a subset of the 8-layers tracks collected from tower 
40 during Run 358395 (August 15-16, 2018) are presented. This was a $13 \mathrm{TeV}$ proton-proton collision run with an average number of interactions per bunch crossing of $\langle\mu\rangle=31$. A total of 859196 events have been processed by the FTK system during this run.

Figure $2 \mathrm{~b}$ shows the number of tracks processed per event. The limited number of tracks is due to the small region covered $(-0.2<\eta<1.2,2.4<\phi<2.8)$.

The 8-layer tracks produced by FTK include the clusters on track, the track $\chi^{2}$, and the pattern number information. To validate the FTK system output tracks, the FTK functional simulation [5] is run on the raw data for a subset of the events. The simulated tracks are then matched to the tracks generated by the FTK system. In the case of Run 358395, 500 events were selected to perform this validation. Figure 3a shows the fraction of simulated tracks matched with the FTK system tracks. The obtained ratio is plotted as a function of the event number, which corresponds to the time from the beginning of the run. The obtained ratio is at the level of $80-90 \%$. By matching the hardware tracks with simulation tracks, checks on the phase space covered by the hardware tracks can be performed. Figure $3 \mathrm{~b}$ reports the transverse momentum distribution of the tracks matched to FTK found tracks.

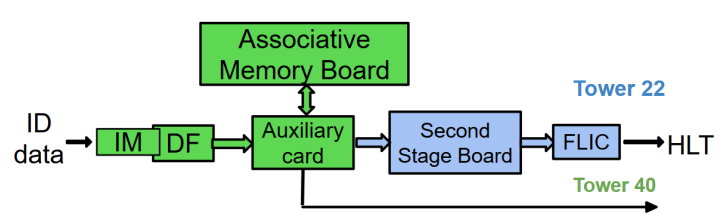

(a)

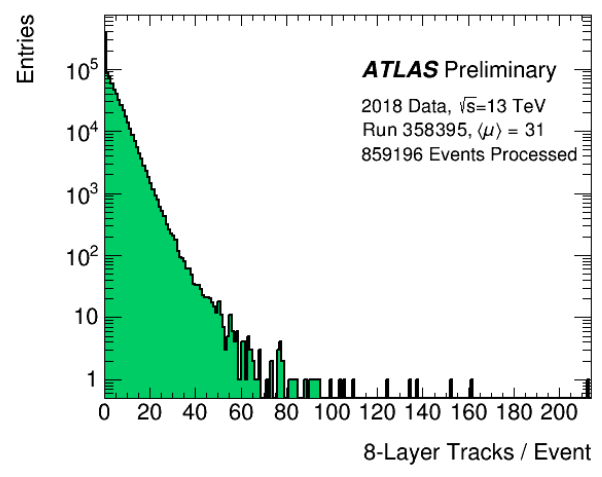

(b)

Figure 2:

(a): schematic representation of the two FTK towers processing pipelines [1] used for system validation. The FTK tower 22 pipeline (green+blue) is a full replica of the FTK system pipeline, it outputs 12-layers tracks. The FTK tower 40 pipeline (green) stops at the Auxiliary Card, it outputs 8-layer tracks.

(b): number of 8-layer tracks per event produced by FTK tower 40 [1]. The plot is based on 859196 events recorded during proton-proton collision at a centre of mass energy of $\sqrt{\mathrm{s}}=13 \mathrm{TeV}$.

\section{Run 3 Configuration}

The 2018 data taking has proven to be a good test bench to validate the FTK-HLT integration. This validation will continue until the end of the LHC Run 2 (December 3, 2018). In this period the FTK will continue commissioning, improve data quality and scale up the system. While the current system configuration allows for performance optimization, the Run 3 configuration will be commissioned during the LHC long shutdown. In this configuration the system will include 128 


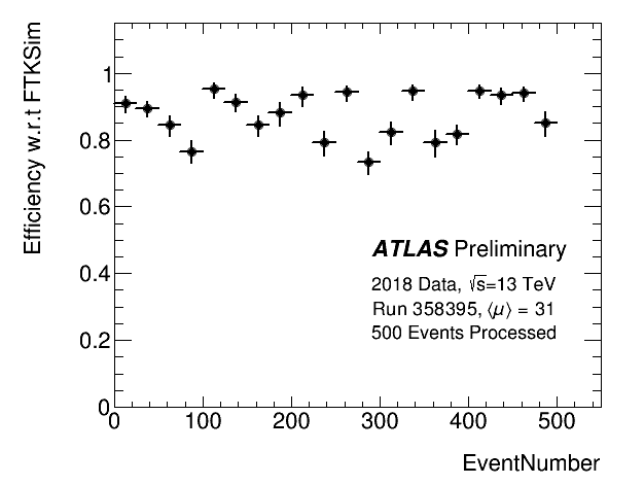

(a)

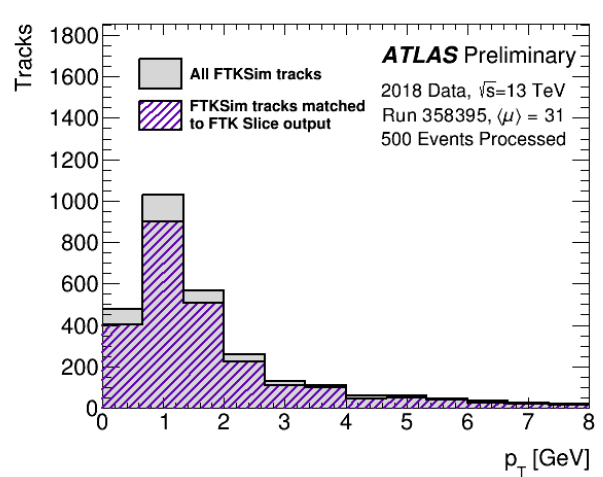

(b)

Figure 3:

(a): fraction of FTK tower output tracks that are matched to tracks produced by running FTK functional simulation [5] on raw data for 500 events of Run 358395 [1].

(b): the he $p_{\mathrm{T}}$ distribution of the tracks in Figure (a) [1]. The distribution of tracks matched between the hardware and simulation (purple stripes) is compared to all simulated tracks for the same events (solid grey).

Processing Units doubling the number of AMB, AUX and SSB boards included in the system. The system validation will proceed during the long shutdown by feeding prerecorded data to the system.

\section{Summary and conclusion}

The FTK system is currently under commissioning and it is producing its first tracks with collision data. A tower of the final FTK system was integrated and tested in ATLAS allowing evaluation of the system performances on real data. We showed the first results on system performance studies. Those measurements will allow optimization of the system improving the performances. The FTK system commissioning will continue during the LHC long shutdown (2019-2020). The full FTK system will be operational by the beginning of Run 3 foreseen by 2021 .

\section{References}

[1] FTK Public Results, https://twiki.cern.ch/twiki/bin/view/AtlasPublic/FTKPublicResults

[2] ATLAS Collaboration, JINST 3 (2008) S08003. doi:10.1088/1748-0221/3/08/S08003

[3] ATLAS Collaboration, Eur. Phys. J. C 77 (2017) no.5, 317 doi:10.1140/epjc/s10052-017-4852-3

[4] M. Shochet et al., CERN-LHCC-2013-007, ATLAS-TDR-021. http://cdsweb.cern.ch/record/1552953

[5] J. Adelman et al., Procedia Comput. Sci. 66 (2015) 540. doi:10.1016/j.procs.2015.11.061

[6] M. Dell'Orso and L. Ristori, Nucl. Instrum. Meth. A 278 (1989) 436. doi:10.1016/0168-9002(89)90862-0

[7] S. Citraro et al., IEEE Trans. Nucl. Sci. 63 (2016) no.2, 1147. doi:10.1109/TNS.2016.2529718

[8] A. Annovi et al., JINST, 12, April 2017, TWEPP2016 doi:10.1088/1748-0221/12/04/C04013 